\title{
Measuring ground displacements from SAR amplitude images : application to the Landers earthquake
}

\author{
Rémi Michel, Jean-Philippe Avouac, \\ Laboratoire de Détection et de Géophysique, CEA,Bruyères le Chatel, France
}

\author{
Jean Taboury \\ Institut d'Optique- SupOptique, Orsay, France
}

\begin{abstract}
ERS SAR amplitude images are utilized to map ground displacements from a sub-pixel correlation method. It yields a ground two-dimensional displacement field with independent measurements about every $128 \mathrm{~m}$ in azimuth and $250 \mathrm{~m}$ in range. The accuracy depends on the characteristics of the images. For the Landers test case, the $1-\sigma$ uncertainty is $0.8 \mathrm{~m}$ in range and $0.4 \mathrm{~m}$ in azimuth. We show that this measurement provides a map of major surface fault ruptures accurate to better than $1 \mathrm{~km}$ and information on coseismic deformation comparable to the 92 GPS measurements available. Although less accurate, this technique is more robust than SAR interferometry and provides complementary information since interferograms are only sensitive to the displacement in range.
\end{abstract}

\section{Introduction}

The measurement of ground displacements is a key issue in seismotectonics. Geodetic techniques need pre-earthquake campaigns and provide accurate but few measurements. Satellite imagery regularly provides detailed and spatially comprehensive images and is a most valuable alternative especially to study remote areas. Satellite radar interferometry (INSAR) has become a popular method for the study of earthquakes [e.g. Massonnet et al., 1993]. There are however some severe limitations to this technique $:$ it is very sensitive to phase decorrelation, the determination of displacements requires phase unwrapping which is often difficult, it only provides one component of the displacement vector and the interferograms are saturated when the gradient of the displacement exceeds half a fringe per pixel. Ground displacements may also be derived from the offsets between two satellite images acquired before and after an earthquake. Offsets reflect the difference in position in the two images of a given point of the ground. In the present paper we show that SAR amplitude images are particularly well suited for this application and we propose a specific method that accounts for the characteristics of SAR images and of coseismic ground displacements. Hereafter we first describe the technique. We then discuss its accuracy and estimation of uncertainties. We finally investigate its potential for the study of coseismic ground displacements during the Landers earthquake. We show that it provides as tight constraints on the fault model as the numerous geodetic data available for this earthquake.

Copyright 1999 by the American Geophysical Union.

Paper number 1999GL,900138.

0094-8276/99/1999GL900138\$05.00

\section{Method for the Determination of Offsets}

Offsets may be determined from a broad range of techniques [Brown, 1992]. The selection of the most suitable technique depends on the characteristics of the images and of the offsets. For large earthquakes $(\mathrm{Mw}>7)$ coseismic deformation is characterized by displacements of a few tens of centimeters and gradients typically lower than $0.1 \%$ except close to surface fault breaks. As the resolution of satellite images are not better than few meters a sub-pixel estimate of offsets is required. Feature tracking and pattern recognition methods [ $\mathrm{Li}$ et al., 1995; Paillou and Gelautz, 1998] are not amenable to accurate and dense sub-pixel measurements. The differential method [Tian and Huhns, 1986] is too sensitive to noise. The method based on the optimization of the interferometric fringes [Gabriel and Goldstein;1988] fails when phase coherence is low. The iterative methods [Djamji, 1993] deal with high gradients but do not allow a sub-pixel accuracy. Offsets can also be determined by stochastic methods [Bernard, 1989]. They require too many computations to be applied in the present study. Correlation methods, [e.g. Schaum and Mc Hugh, 1991], are most appropriate provided that the displacement field can be modeled locally by a translation that varies smoothly over several pixels. The two components of the translation can be derived from the local cross-correlation peak. The accuracy of correlation methods is limited by the pixel size, or more precisely by the width of the autocorrelation peak of the images. It may also suffer from limitations due to the stereoscopic effect and decorrelation (as radiometric noise). They are not very efficient for SPOT images which often include wide homogenous areas that result in a poor correlation. Though their spatial resolution is roughly similar to that of SPOT images, the ubiquitous presence of speckle in SAR images results in a sharp auto-correlation peak that may yield a sharp correlation peak provided that the speckle patterns are similar in the two images. SAR images with as small as possible baseline (distance between the two orbits) are therefore chosen in order to minimize the stereoscopic effects and speckle geometrical decorrelation. The correlation is computed between two sliding windows, $a$ and $b$, extracted from the images. If $a$ and $b$ are translated by an amount $u$ in range (columns) and $v$ in the azimuth (lines), their Fourier transforms $\tilde{a}$ and $\vec{b}$ verify:

$$
\tilde{p}=\frac{\tilde{a} \cdot \tilde{b}^{*}}{|\tilde{a} \cdot \tilde{b}|}=e^{2 \pi j(u \mu+v v)}
$$

where $\mu$ and $v$ are the coordinates in the Fourier domain. The inverse Fourier transform $p$ of $\tilde{p}$ is the symmetrical Phase Only Filter correlation function [Kotynski and Chalasinska- 


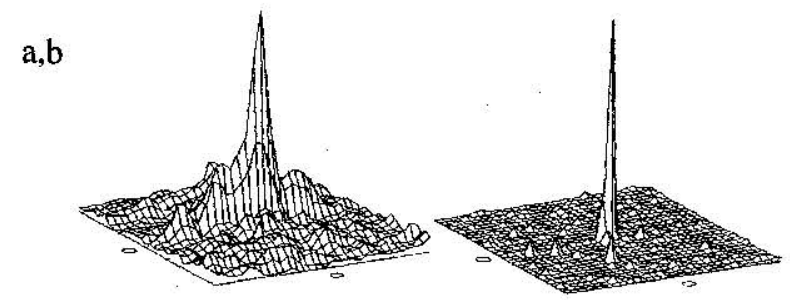

Figure 1. Comparison between classical (a) and Symmetrical Phase Only Filter (b) correlation peaks, computed on two $32 \times 32$ windows (from ERS SCL images orbit/frame: $5554 / 2907$ and $10063 / 2907$ ). Axis are 32 pixels long. The Scales pixel size is about $4 \mathrm{~m}$ in azimuth and $7.8 \mathrm{~m}$ in range. The much narrower and less noisy correlation peak obtained in b) allows determination of offsets with a sub-pixel accuracy (0.01 pixel in that case).

Macukow, 1996] which is maximum at $(u, v)$. The normalization in eq. 1 is a non-linear filtering of the classical correlation function defined in the Fourier domain as $\tilde{a} \tilde{b}^{*}$. This normalization is possible with SAR images as $\left.\right|^{a b} \mid$ is never small due to speckle. It can not be applied to SPOT images. It yields directly the phase difference between $\vec{a}$ and $\vec{b}$ hence the translation. Fig. 1 shows that, contrary to the classical correlation, eq. 1 leads to a sharp peak allowing for a sub-pixel determination of $(u, v)$. We have tested various window sizes and found that in the present study $32 \times 32$ pixels is well adapted. It allows a dense coverage of independant measurements and is consistent with the low gradient hypothesis mentioned above. $(u, v)$ is derived from the barycenter of $p$ around a $3 \times 3$ pixcls vicinity of the maximum. An estimate of the signal to noise ratio, $S N R$, on that measurement is the ratio of the energy within the $3 \times 3$ vicinity of the maximum and the energy outside this vicinity [Schaum and Mc Hugh, 1991]. A low SNR may result from temporal decorrelation due to changes in the radiometry, local high gradients due to topography or non overlapping areas of $a$ and $b$. To reduce this latter effect a coarse estimate is first computed with a pixel accuracy. The window $b$ is then extracted with account for this estimate and a precise measurement is performed. The effect of topography is reduced by choosing small baselines.

\section{Ground Displacements Derived from Offsets}

Offsets in range $(u)$ depend on ground displacement, on the stereoscopic effect and on the differences in range pixel size $(\sim 7.8 \mathrm{~m}$ for ERS SCL images [ESA, 1992]). Provided that a Digital Elevation Model is available, the stereoscopic contribution $u_{\text {sopo }}$ can be computed [Lebert, 1990]. The residual term $u$ - $u_{\text {iopo }}$ is the component in range of the ground displacement, that can also be derived by unwrapping interferograms. Offsets in azimuth $(v)$ depend on the ground displacement, on a stereoscopic like effect due to non parallel orbits and different Doppler centroid frequencies, and on the differences in azimuth pixel size ( $\sim \mathrm{m}$ for ERS Single Look Complex images [ESA, 1992]). The stereoscopic effect is minimized by using images processed with the same Doppler frequency and the effect of pixel size is compensated from the characteristics of the images. We have analyzed uncertainties using two ERS-1 SLC images including no ground deformation. The ground displacement retrieved from the offsets should therefore reflect errors of the measurements. In that case the contribution of the topography is less than 0.01

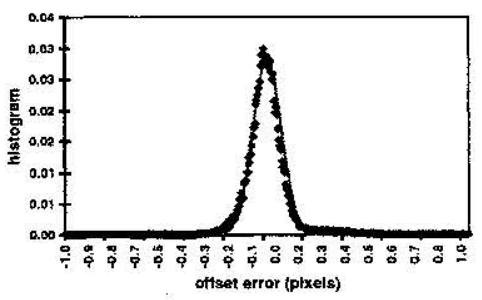

Figure 2. Distribution of ground displacements derived from two ERS1 SLC images (o/f : 5554/2907 and 10063/2907). True ground displacement is zero so this histogram shows the distribution of errors. 34000 independent measurements in range and in azimuth are plotted together (dots) expressed in pixel size. The best fitting gaussian distribution (continuous line) is centered on $10^{-6}$ pixel and has a variance of 0.1 pixel.

pixel and was not corrected. We calculated the ground displacement and the $S N R$ as described above to yield the results shown in Fig.2 and Fig.3. The 34000 independent measurements displacements indicates a gaussian distribution with an average of $10^{-6}$ pixel and a variance of 0.1 pixel (Fig.2). Thus the ground displacements measured by our technique are not significantly biased and the $1-\sigma$ uncertainty on individual measurement, in that case, is 0.1 pixel on average. This value would actually depend on the images. Fig.3 further indicates that the errors are strongly correlated with $S N R$ so that $S N R$ may be used to estimate the $1-\sigma$ uncertainty associated with each individual measurement.

\section{Application to the Landers Earthquake}

The Landers earthquake was chosen to evaluate the potential of offsets because it was extensively surveyed. In particular Hudnut et al. [1994] have provided a model (Hudnut et al's model) of coseismic deformation that accounts for the 92 available GPS and for an extensive mapping of fault breaks (Fig.4a). This model consists of 27 elementary dislocations and was shown to be consistent with the differential interferograms obtained by Massonnet et. al. [1994] and does not differ significantly from those derived from seismological observation [ Hernandez et al., 1997].

\subsection{Measurements of Ground Displacements}

The ground displacement were derived from two ERS-1 SLC images taken before and after the earthquake (Fig.4b). The correlation window is $32 \times 32$ pixels in size leading to independent measurements every $250 \mathrm{~m}$ in range and $128 \mathrm{~m}$ in azimuth. The average uncertainty is $1 / 10^{\text {th }}$ of the pixel size corresponding to $0.8 \mathrm{~m}$ in range and $0.4 \mathrm{~m}$ in azimuth. The

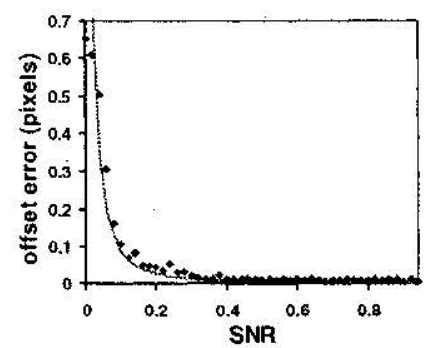

Figure 3. Relation between Signal to Noise Ratio (SNR) and measurement errors (in pixel) obtained by computing the standard deviation of all measurements corresponding to a given value of $S N R(+-0,01)$. Same data as for Fig.2. The continuous line is an estimate of $1-\sigma$ as a function of $S N R$. 

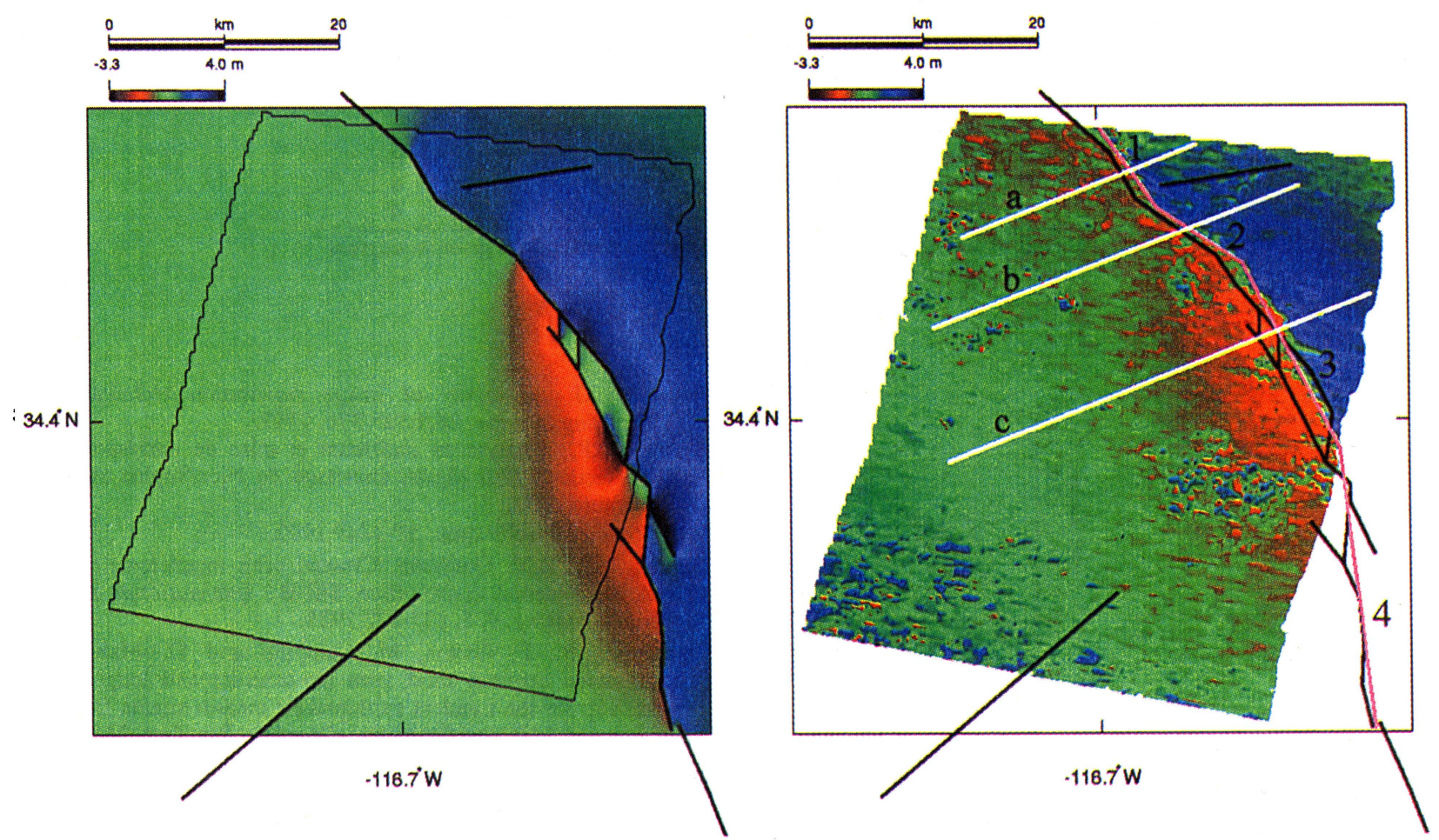

Figure 4. Displacement field in azimuth computed from the model of Hudnut et al [1994] (a) and measured in this study (b) using the ERS-1 SLC images (o/f : $0554 / 2907$ and 4051/2907, UTM cartographic projection). It consists of 34000 independent measurements about every $128 \mathrm{~m}$ in azimuth and $250 \mathrm{~m}$ in range. Blacks lines depict the 27 faults considered by Hudnut et al.[1994]. Pink lines depict a simplified model with 4 faults (see text).

ground deformation in range is below the noise level but measurements in azimuth show a clear signal (Fig 4b) indicating right lateral slip along a discontinuity that coincides within $1 \mathrm{~km}$ with the Hudnut et al's model. In addition, Fig.4 and 5 show that our measurements are roughly consistent with the displacements computed from the Hudnut et al's model hence with the 92 GPS data used by Hudnut et al. [1994]. Fig. 5 does however show systematic misfits larger than the 1$\sigma$ uncertainty on the measured offsets. Those misfits may have a physical basis. For example they seem to occur systematically in area where the elastic dislocation model would predict particularly large strain possibly above the elastic threshold of the uppermost brittle crust. Another explanation is that Hudnut et al's model was essentially constrained from measurements relatively far from the faults zone. It may therefore predict rather poorly constrained estimates of the displacement field in the near-source area covered by the SAR images. The regular oscillations in the profile of Figure $5 \mathrm{~b}$ and $5 \mathrm{c}$ may be artifacts due to the SAR processor. The large one between 20 and $30 \mathrm{~km}$ coincides with an area of particularly low correlation.

\subsection{Assessing the Information Available from Offsets}

We discuss now the fault model that could be derived from the offsets considered as the only source of information as it would be the case for an earthquake in a remote area. The contribution of the faults lying outside the area covered by the SAR images is not analyzed. For simplicity they were replaced by a single dislocation (dislocation $\mathrm{n}^{\circ} 4$ in Table.1). Fig $4 \mathrm{~b}$ would suggest a fault model consisting of 3 other segments. Displacements are computed from the analytical formulation of Okada [1985]. Direct comparison between offsets and GPS is not possible because among the 92 GPS measurements available for that earthquake, only 6 lies within the area where offsets were measured. This is why we compare in Figure 6 the GPS measurements with displacements computed from a fault model derived from the offsets. We used an iterative linearized least-squares approach to invert the parameters of the sources starting with vertical faults. The estimated parameters are the two components of the slip vectors and faults depth (Table 1). The slips and the depth are obtained with an uncertainty of respectively $24 \mathrm{~cm}$ and $2 \mathrm{~km}$. The model
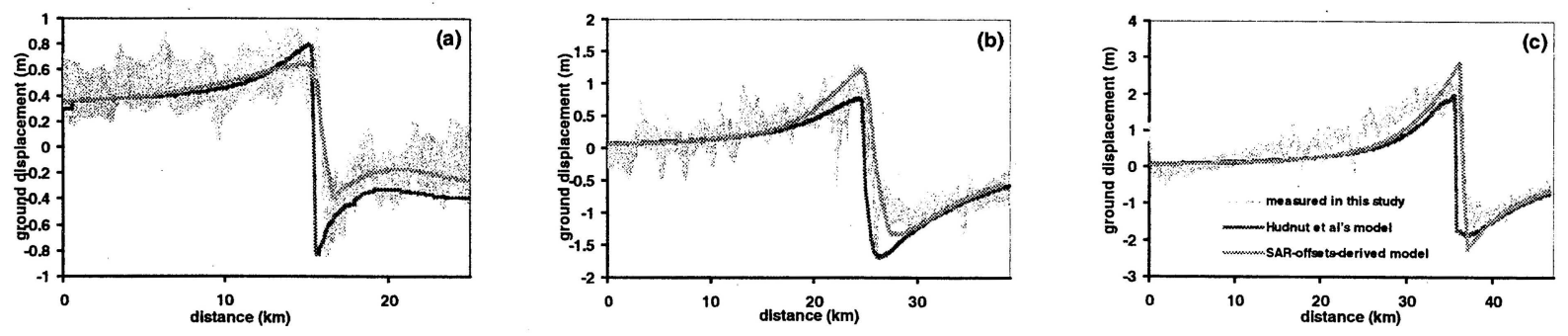

Fig.5. Comparison between displacements in azimuth 1) measured from offsets (1- $\sigma$ error bars), 2) computed from the Hudnu tet al's model and 3) computed from the SAR_offsets_derived model. See Fig. 4 for the profiles. 
Table 1. Fault model derived from $S A R$ offsets

\begin{tabular}{cccccccc}
$\begin{array}{c}\text { Fault } \\
\text { segment }\end{array}$ & $\begin{array}{c}\text { latitude } \\
\left({ }^{\circ} \mathrm{N}\right)\end{array}$ & $\begin{array}{c}\text { longitude } \\
\left({ }^{\circ} \mathrm{E}\right)\end{array}$ & $\begin{array}{c}\text { length } \\
(\mathrm{km})\end{array}$ & $\begin{array}{c}\text { azimuth } \\
\left({ }^{\circ} \mathrm{E}\right)\end{array}$ & $\begin{array}{c}\text { slip } \\
(\mathrm{m})\end{array}$ & $\begin{array}{c}\text { depth } \\
(\mathrm{km})\end{array}$ \\
\hline 1 & $34.652^{\circ}$ & $-116.680^{\circ}$ & 11.28 & $-39.6^{\circ}$ & 2.33 & 5 \\
2 & $34.587^{\circ}$ & $-116.627^{\circ}$ & 8.60 & $-54.4^{\circ}$ & 2.58 & 8 \\
3 & $34.479^{\circ}$ & $-116.518^{\circ}$ & 20.95 & $-21.0^{\circ}$ & 6.22 & 4 \\
4 & $34.284^{\circ}$ & $-116.450^{\circ}$ & 25.10 & $-4.0^{\circ}$ & 2.76 & 9
\end{tabular}

All fault planes are vertical and reach the surface. Angle are given in degrees , azimuth is given counterclockwise from due North.
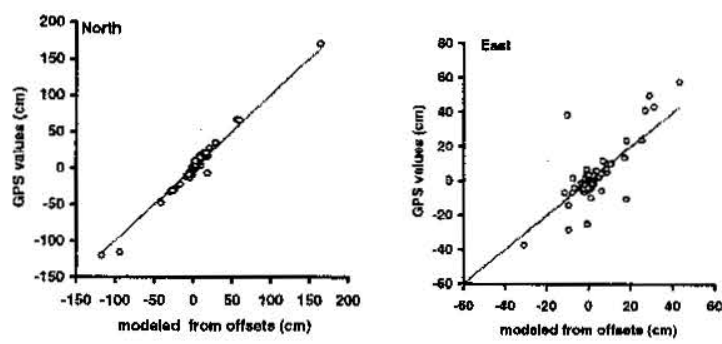

Figure 6. Comparison between GPS measurements and predicted displacements computed from the SAR_offsets_derived. Continuous lines show $1: 1$ ratios. The $\mathrm{rms}$ error is $4.8 \mathrm{~cm}$ in horizontal and $10.5 \mathrm{~cm}$ in vertical.

( $S A R \_$offsets_derived_model) fits the measurements with a residual standard deviation of $49 \mathrm{~cm}$ which is comparable with the average measurements uncertainty of $40 \mathrm{~cm}$. Fig. 6 shows that this simple fault model accounts satisfactorily for the measured displacements. The standard deviation of the misfit is $4.5 \mathrm{~cm}$ for the horizontal displacements and $10.5 \mathrm{~cm}$ for the vertical displacements. For comparison the rms misfits between the GPS measurements and the displacements computed from the Hudnut et al's model are respectively 4.1 $\mathrm{cm}$ and $9.2 \mathrm{~cm}$. It thus appears that the $S A R \_$offsets_derived_model does fit the GPS data nearly as well as the much more constrained model proposed by Hudnut et al. [1994]. It indicates that offsets provide an information comparable to the much more accurate GPS measurements available for this earthquake. This is because the relatively poor accuracy of offsets is balanced by the large amount of data in the near-source area, and the dense spatial coverage. This demonstrates the potential of this technique for the study of future earthquakes for which SAR interfometry or preearthquake geodetic survey would not be available.

\section{References}

Bernard, S.T., Stochastic stereo matching over scale, Int. J. of Comp. Vision, 3,17, 1989.

Acknowledgments. We are most grateful to Jean-Bernard de Chabalier and Gilles Peltzer for fruitful discussions.
Brown, L.G., A survey of image registration techniques, $A C M$ Computing Surveys, 24,4,325-376, 1992.

Djamdji, J.P., Analyse en ondelettes et mise en correspondance en télédétection, PhD thesis, université de Nice-Sophia Antipolis, 2 Décembre 1993.

ESA, Product Specification, SP-1149,1992.

Gabriel, A.K. R.M. Goldstein, Crossed orbit interferometry : theory and experimental results from SIR-B, International Journal of Remote Sensing, 9, 8, 857-872,1988.

Hernandez, B., F. Cotton, M. Campillo and D. Massonnet, A comparison between short term (co-seismic) and long term (one year) slip for the Landers earthquake : measurements from strong motion and SAR interferometry, Geophys. Res. Lett, 24, 13, 1579$1582,1997$.

Hudnut, K.W. and 16 others, Coseismic displacements of the 1992 Landers earthquake sequence, Bull. Seism. Soc. Am., 84, 625-645, 1994.

Kotynski, R., and K. Chalasinska-Macukow, Optical correlator with dual non-linearity, Journal of Modern Optics, 43, 2, 295-310, 1996.

Leberl, F., Radargrammetric image processing, Artech House, Nonwood, MA, 1990.

Li, H., B.S. Manjunath, and S.K. Mitra, A contour-based approach to multisensor image registration, IEEE Transaction on Image Processing, 4, 3, 320-334, 1995.

Massonnet, D., M. Rossi, C. Carmona, F. Adragna, G. Peltzer, K. Feigl and T. Rabaute, The displacement field of the Landers earthquake mapped by radar interferometry , Nature, 364, 138-142, 1993.

Okada,Y., Surface deformation due to shear and tensile faults in elastic half-space, Bull. Seism. Soc. Am., 75, 1135-1154, 1985.

Paillou, Ph. and M. Gelautz, The optimal gradient matching method: application to X-SAR and Magellan Stereo images, Proceedings of IGARS''98, 1998.

Schaum, A and M. McHugh, Analytic method of image registration : displacement estimation and resampling, Report of the Naval Research Laboratory, NRL Report n ${ }^{\circ}$ 9298, 1991.

Tian, Q., and M.N., Huhns, Algorithms for subpixel registration, Computer Vision, Graphics, and Image Processings, 35, 220-233, 1986 .

J.P. Avouac and R. Michel, Laboratoire de Détection et de Géophysique, CEA, BP 12, 91680 Bruyères le Chatel Cedex, France. (e-mail: michel@Idg.bruyeres.cea.fr)

J. Taboury, Institut d'Optique, 91403 ORSAY Cedex, France.

(Received August 17, 1998; revised January 20, 1999; accepted February 8, 1999.) 